\title{
Electrophoretic Deposition of Zirconia Nanoparticles
}

\author{
Stefan Frank ${ }^{1}$, Carolina Mochales ${ }^{1 *}$, Martin Heimann ${ }^{1}$, Felix Kochbeck ${ }^{1}$, Rolf \\ Zehbe $^{2}$, Claudia Fleck $^{2}$ and Wolf-Dieter Mueller ${ }^{1}$ \\ ${ }^{1}$ Dental School, Charité Universitaetsmedizin Berlin, Germany \\ ${ }^{2}$ Materials Engineering, Berlin Institute of Technology, Germany
}

Received: December 13, 2013; Accepted: February 10, 2014; Published: February 12, 2014

“Corresponding author: Carolina Mochales, Dental School, Charité Universitaetsmedizin Berlin, 14197 Berlin, Germany, Tel: +49-30-450-562756; Fax: +49-30-450-562923; E-mail: carolina.mochales-palau@charite.de

\begin{abstract}
Zirconia-based ceramics have gained considerable interest for several applications (e.g. solid electrolytes in fuel cells and in oxygen sensors, thermal barrier coatings and biomaterials for dental and orthopedic applications) due to their high mechanical strength, improved fracture toughness and easy affordability. Generally, one of the main disadvantages of ceramic materials is their brittleness, i.e. low fracture toughness. Electrophoretic deposition (EPD) has recently gained interest in the ceramics processing due to its wide versatility, allowing the fabrication of laminated and graded ceramics, fiberreinforced ceramic matrix composites, coatings, and nanostructures. Moreover, EPD has been pointed out as an effective technique to obtain complicated 3D shape structures. This makes EPD a promising technique to obtain ceramic constructs for several applications like solid oxide fuel cells, thermal barrier coatings and biomaterials. In our research group, we have developed both a manual and automated EPD set-up to obtain tetragonal and cubic zirconia multilayered ceramic constructs. Multi layers of alternating tetragonal and cubic phases with a clearly defined interface were successfully obtained by an EPD layering process with submicron sized powders of Y-TZP with different mol percentages of yttrium oxide (3\% and $8 \%$ ). Finally, we investigated how to obtain 3D shape structures by EPD and we applied this technique to the dental materials field in order to obtain dental crowns.
\end{abstract}

Keywords: Electrophoretic deposition; Nanoparticles; Multi layered ceramics; Dental crowns

\section{Introduction}

Zirconia occurs in three crystal modifications at low-pressure conditions: monoclinic, tetragonal and cubic. The monoclinic phase is stable up to $1170^{\circ} \mathrm{C}$, while the tetragonal phase is stable between $1170^{\circ} \mathrm{C}-2370^{\circ} \mathrm{C}$, and the cubic one above $2370^{\circ} \mathrm{C}$ [1].

Doping of zirconia with dopants like $\mathrm{CaO}, \mathrm{MgO}, \mathrm{CeO}_{2}$ and $\mathrm{Y}_{2} \mathrm{O}_{3}$ results in stabilization at room temperature of the tetragonal phase at dopant concentrations below $\sim 8 \mathrm{~mol} \%$ and in stabilization of the cubic phase at concentrations above $\sim 8$ mol\%. The stabilization of the tetragonal phase results in a remarkable increase in mechanical toughness, whereas the stabilization of the cubic phase results in an increase of the ionic conductivity to values significantly higher than for other ceramics
$[1,2]$. This high ionic conductivity of the cubic zirconia makes it a very suitable material as electrolyte for solid oxide fuel cells (SOFCs). While tetragonal zirconia polycrystals (TZP) are being used in the dental and orthopedic field due to their relatively high toughness [3]. Moreover, the use of Yttria stabilized ceramics as thermal barrier coatings (TBCs) is of big importance [4].

Electrophoretic deposition is essentially a two-step process. In the first step, charged particles suspended in a liquid migrate towards an electrode under the effect of an electric field (electrophoresis); while in the second step, the particles deposit on the electrode forming a relatively dense and homogeneous compact or film. A post-EPD processing step is usually required, which includes a suitable heat-treatment (firing or sintering) in order to further densify the deposits and to eliminate porosity [5-7].

The interest in electrophoretic deposition (EPD) for nanomaterials and ceramics production has widely increased due to the versatility of this technique to effectively combine different materials in unique shapes and structures, like laminated and graded ceramics, fiber-reinforced ceramic matrix composites, coatings, and nanostructures [8-12]. Moreover, EPD has been pointed out as an effective technique to obtain complicated 3D shapes [13,14].

In our research work, we have started an EPD laboratory where we are able to produce several ceramic structures like multilayered specimens and 3D shape structures.

For instance, in order to optimize the properties of the final ceramic by combining the high mechanical toughness of the tetragonal phase of zirconia together with the high ionic conductivity of its cubic phase, we recently produced tetragonal and cubic zirconia multilayered specimens with a sharp and well inter connected interface by electrophoretic deposition of nano sized powders of Y-TZP with different mol percentages of yttrium oxide (3\% and $8 \%$ ) $[15,16]$.

Finally, we also investigated the EPD of 3D shapes and we applied this technique to obtain ceramic dental crowns. 


\section{Methods}

\section{Samples preparation}

EPD suspensions: The wide range of different zirconia multilayered samples as well as 3D shaped samples needed several varied suspensions. Two different $\mathrm{Y}_{2} \mathrm{O}_{3}$-stabilized $\mathrm{ZrO}_{2}$ powders (TZ-3Y with 3 mol\% $\mathrm{Y}_{2} \mathrm{O}_{3}$, and TZ-8Y with $8 \mathrm{~mol} \% \mathrm{Y}_{2} \mathrm{O}_{3}$ ) from Tosoh (Shunan, Japan) were used for the experimental investigations. According to the data given by the manufacturer the used powder contained $5.22 \mathrm{wt} \% \mathrm{Y}_{2} \mathrm{O}_{3}$ for TZ-3Y, $13.60 \mathrm{wt}$ $\% \mathrm{Y}_{2} \mathrm{O}_{3}$ for TZ-8Y. The specific surface areas and crystallite sizes were $14.8 \mathrm{~m}^{2} / \mathrm{g}$ and $26 \mathrm{~nm}$ for TZ-3Y, $13.5 \mathrm{~m}^{2} / \mathrm{g}$ and $23 \mathrm{~nm}$ for TZ-8Y.

All solutions were prepared with ethanol (Merck KGaA, Darmstadt, Germany). In order to adjust the suspension's $\mathrm{pH}$ either acetic acid (Carl Roth GmbH \& Co. KG, Karlsruhe, Germany) or benzoic acid (Sigma Aldrich, St. Louis, USA) was added to the suspension. To produce the 3D shaped (i.e. dental crowns) a polymer was added to the TZ- $3 \mathrm{Y}$ solution to increase the flexibility of the final deposit. Prior to use, the suspensions were magnetically stirred for 45 minutes, followed by ultrasonic for at least $3 \mathrm{~min}$, following for further 15 minutes of slow magnetic stirring.

EPD procedure: Different EPD set-ups were used to obtain the different samples showed in this paper. Figure 1 shows schemes of the three set-ups used: a manual (Figure 1a) and an automated (Figure 1b) set-up to produce tetragonal and cubic zirconia multilayered specimens, and a manual set-up to produce dental ceramic crowns (Figure 1c).

For the multilayered samples, aluminium electrodes were used in the manual set-up with a relative distance of $2.5 \mathrm{~cm}$. The deposition area was around $25 \mathrm{~mm}-30 \mathrm{~mm}$ by $10 \mathrm{~mm}$. The electrodes were fixed vertically in the manual set-up, while in the automated set-up they were fixed horizontally. For the automated set-up, the deposition area was $25 \times 45 \mathrm{~mm}$ and the distance between electrodes was $3 \mathrm{~cm}$. In the case of the dental crowns, a modification of the methods described in the literature
$[17,18]$ was used. A plaster stump of a tooth was prepared by typical dentistry techniques and silver painting was applied directly on the plaster stump to create the deposition electrode. The counter electrode was made out of some metallic sheet (e.g. aluminium).

The EPD process of the multilayered samples was carried out at a constant DC voltage of $15 \mathrm{~V}$ for the TZ-3Y layers and of $10 \mathrm{~V}$ for the TZ-8Y layers. TZ-3Y and TZ-8Y layers were deposited consecutively with a fixed deposition time of $30 \mathrm{~min}$ for each layer for the manual set-up (Figure 1a), whereas for the automated setup it was between $1 \mathrm{~min}$ and $5 \mathrm{~min}$. For the dental crowns, only one single layer of tetragonal zirconia (TZ-3Y) was deposited at either $15 \mathrm{~V}$ other $30 \mathrm{~V}$ in periods of time between $10 \mathrm{~min}$ and 20 min.

Final sintering: After drying and removal from the electrode, the deposited multilayered and 3D shaped single layer specimens (i.e. dental crowns) were sintered at $1450{ }^{\circ} \mathrm{C}$ for $3 \mathrm{~h}$ in a VITA Zyrcomat oven (Vita Zahnfabrik, Bad Saeckingen, Germany).

\section{Samples characterization}

The obtained zirconia specimens were investigated via optical microscopy using a regular microscope (Zeiss Axiophot, Carl Zeiss GmbH, Jena, Germany) and the InfiniteFocus ${ }^{\circledR}$ G4 microscope (IFM) (Alicona Imaging, Grambach/Graz, Austria).

The microstructure of the multilayered samples (cubic and tetragonal) and specifically the interface between the layers was analyzed using electron microscopy (SEM; FEI Quanta 600 FEG, FEI, Eindhoven, The Netherlands). To evaluate the yttriadistribution over the interface, energy dispersive X-ray (EDX) analysis was performed with an SEM S-2700 (High-Technologies Co., Japan) equipped with an EDX system (remX GmbH, Bruchsal, Germany) with a Si(Li) detector and a Moxtek AP 1.3 window (active area10 $\mathrm{mm}^{2}$ ). The EDX-measurements were performed at $20 \mathrm{kV}$ and a working distance between the detector and the sample of $17 \mathrm{~mm}$. Moreover, the fractured surface of a multilayered specimen subjected to four point bending test (4PBT) was observed by SEM.
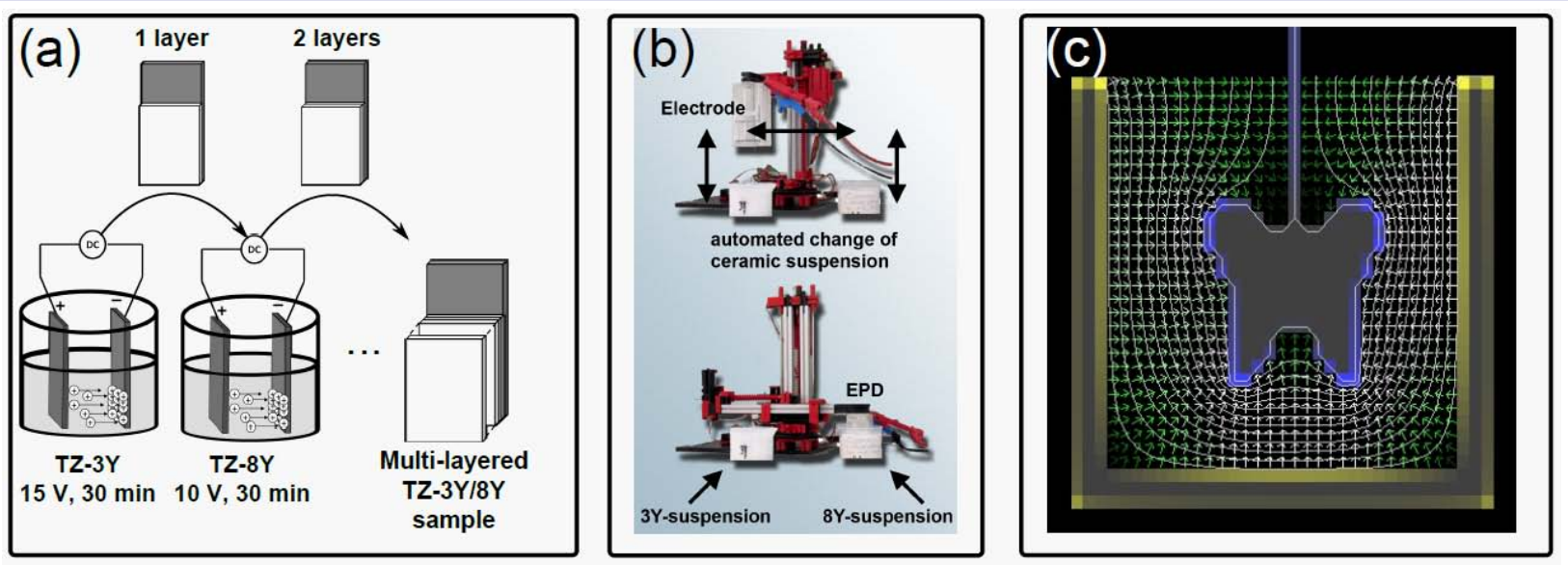

Figure 1: Schematic representation of the EPD set-ups used to produce: tetragonal and cubic zirconia multilayered ceramic specimens, both manually (a) and automatically (b), and tetragonal zirconia, dental crowns (c). 


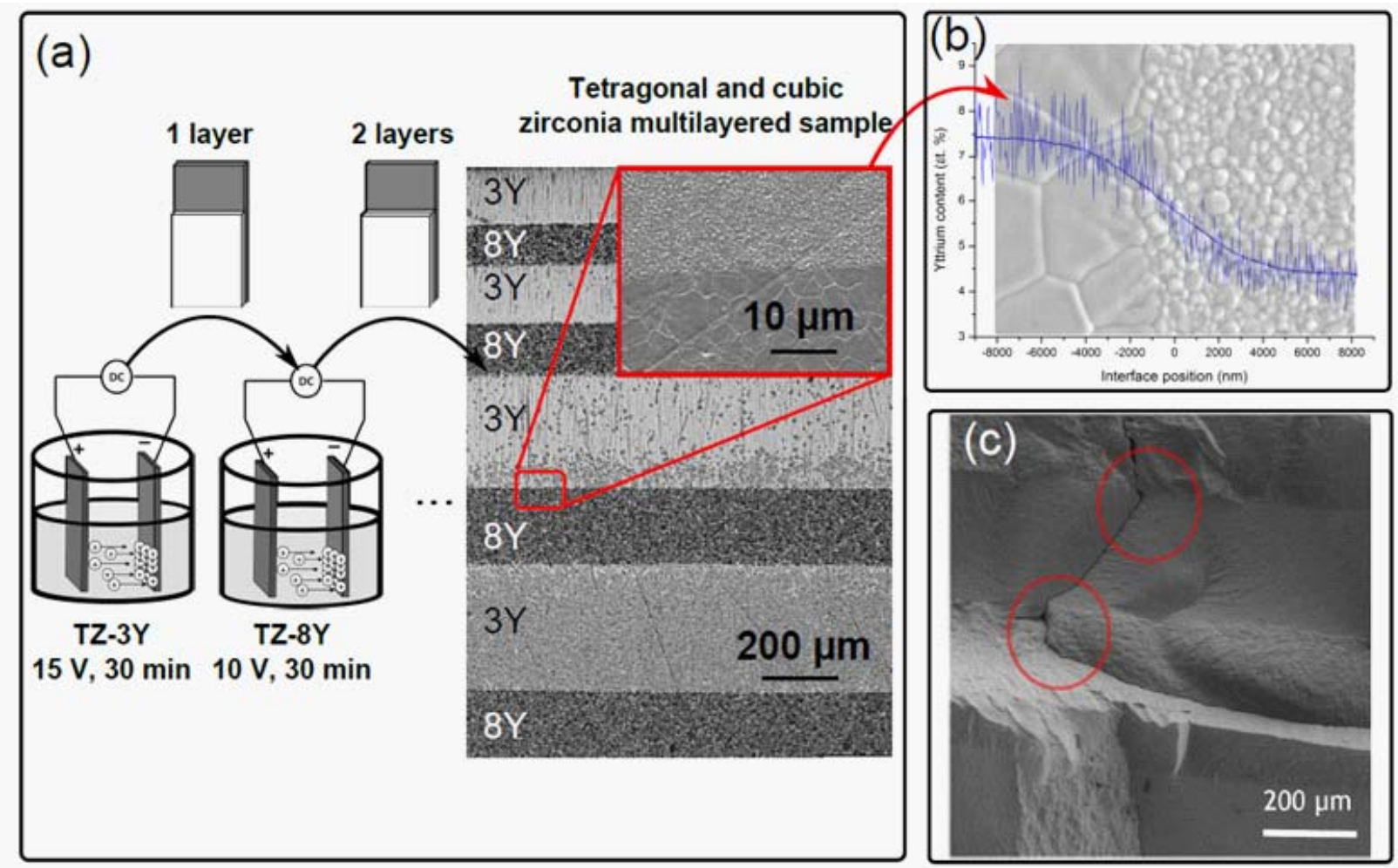

Figure 2: Tetragonal and cubic zirconia multilayered ceramic specimen manually produced by EPD (a), with an inset showing a SEM micrograph of the interface microstructure, which is magnified and showed together with the yttrium wt\% content along the interface (b); SEM micrograph of a fractured surface after 4 point bending test (c).
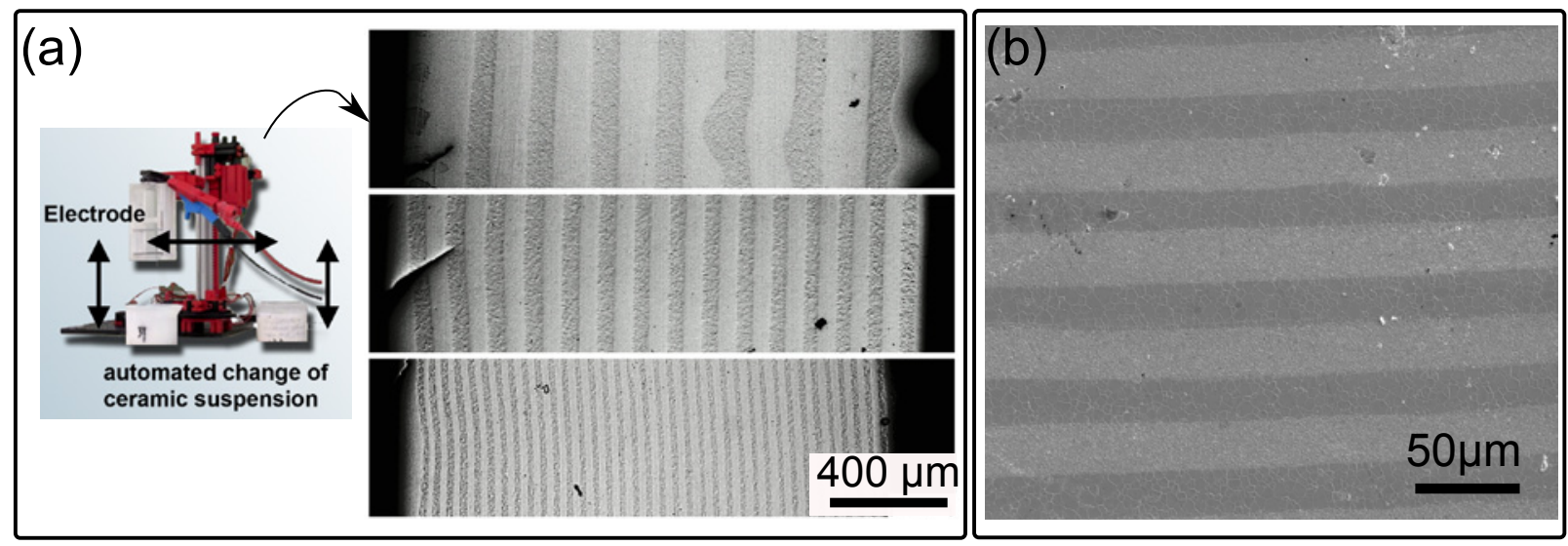

Figure 3: Tetragonal and cubic zirconia multilayered ceramic specimens with different layers thickness produced by an automated EPD set-up (a), with a magnification showing the different microstructure between the tetragonal and the cubic layers (b).

\section{Results and Discussion}

Figure 2 shows a summary of the main characteristics of the tetragonal and cubic multilayered ceramic specimens obtained by using the manual set-up. Figure 2a shows one of the several obtained zirconia multilayered specimens. The used EPD process with constant deposition times for each layer resulted in several multilayered specimens with layers of different thicknesses between 12216 and $142 \mu \mathrm{m}$ for the TZ-3Y, and between 383-139 $\mu \mathrm{m}$ for the TZ-8Y. The SEM observations (inset in Figure 1a, and Figure $2 \mathrm{~b}$ ) revealed a sharp and well inter connected interface between two different microstructures between layers. These well differentiated microstructures between layers were mainly tetragonal and cubic. EDX analysis (Figure 2b) confirmed a change in the corresponding yttrium, zirconium and oxygen amounts of the respective cubic and tetragonal layers. Indeed, this result is in accordance to the $\mathrm{ZrO}_{2}-\mathrm{Y}_{2} \mathrm{O}_{3}$ equilibrium phase diagram as expected [19], as it is the yttrium which rules all the different 

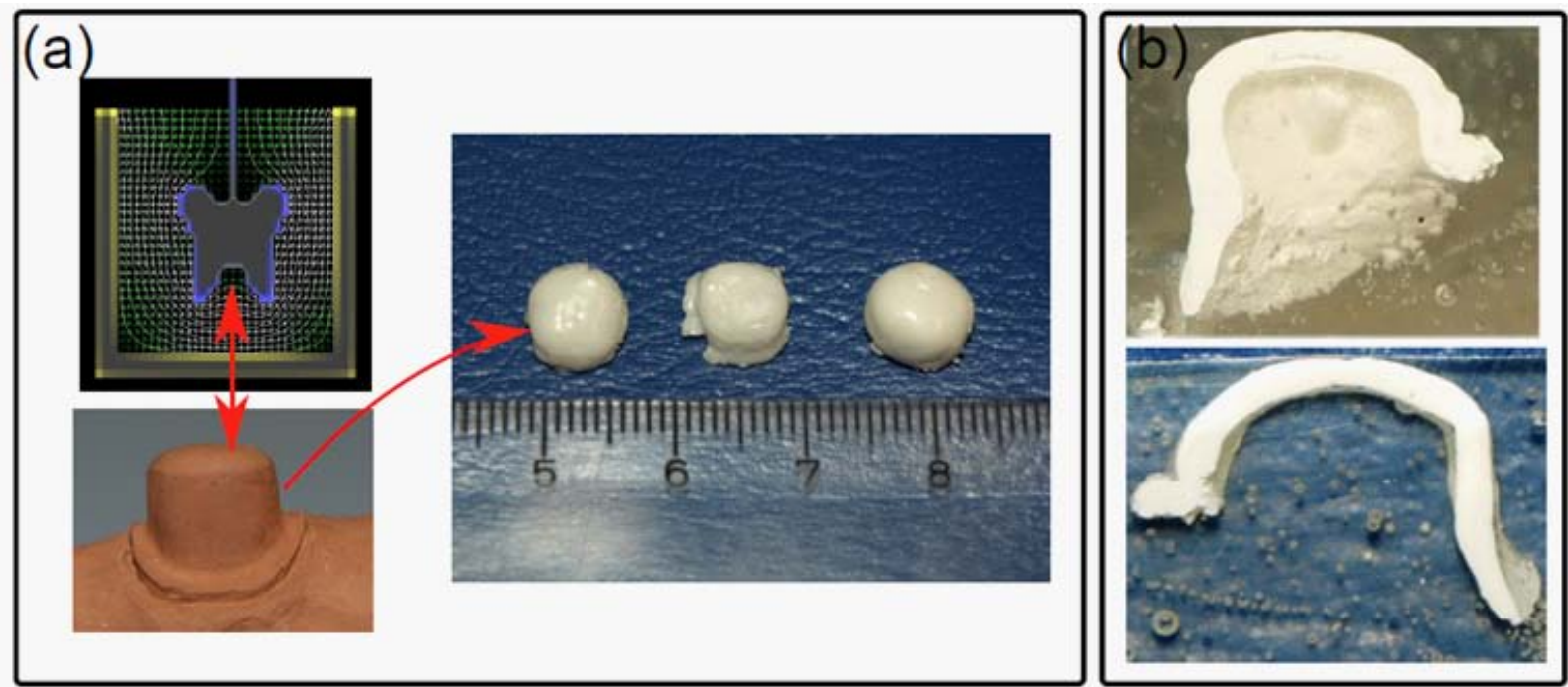

Figure 4: Overview of the EPD set-up, the plaster stump and the dental cap crowns obtained (a), with a transversal cut and a slice of the cap (b).

phase transformations in either tetragonal or cubic zirconia. Figure 2c shows the SEM inspection of the fractured surface of a multilayered specimen subjected to 4-PBT, which revealed significant crack deflection present on the interface between the TZ-3Y and TZ-8Y layers (red circles in Figure 2c). A more detailed discussion of the layers thickness evolution, microstructure and crack deflection in this EPD obtained tetragonal and cubic multilayered ceramic specimens can be found in a previous publication [15].

The production of much thinner layers was possible with the automated set-up, as can be seen in Figure 3. The automation of the EPD process facilitated the operating of the process and ensured a better reliability of the ceramic specimens. Moreover, the in-situ recording of the applied voltage, electric current and temperature during the whole EPD process was used to investigate the electric current evolution with deposition time for the several different layers obtained. More information about the automated set-up and the in-situ recording of the electrical parameters can be seen in a previous work of our group [16].

Figure 4 shows the ceramic dental crowns produced by EPD. An overview of the plaster stump used as deposition electrode and the obtained deposited caps can be seen in Figure 4a. To check the deposited layer in the cap shape we investigated a transversal cut and a slice of the cap (Figure $4 \mathrm{~b}$ ).

These results show that it is possible to obtain optimal ceramic deposits which present different hierarchical levels, i.e. different shape, layers, microstructures and dopant amount, which make these ceramic constructs promising candidates for several applications like solid oxide fuel cells, thermal barrier coatings and biomaterials.

\section{Acknowledgment}

The authors acknowledge the financial support of the DFG (Deutsche Forschungsgemeinschaft) for our project within
SPP1420 (http://spp1420.mpikg.mpg.de/projects/hierarchy-ofmicrostructural-features-as-the-origin-of-fracture-resistance-indentine-and-ceramic-composites).

\section{References}

1. Graeve OA (2008) Zirconia. In: Schakelford JF \& Doremus RH (Eds.), Ceramic and glass materials: structure, properties and processing. Springer, New York, USA, pp. 169-197.

2. Bocanegra-Bernal MH, De la Torre SD (2002) Phase transitions in zirconium dioxide and related materials for high performance engineering ceramics. J Mater Sci 37(23): 4947-4971.

3. Piconi C, Maccauro G (1999) Zirconia as a ceramic biomaterial. Biomaterials 20(1): 1-25.

4. Goswami B, Sahay SK, Ray AK (2004) Application of thermal barrier coatings on combustion chamber liners - A review. High Temperature Materials and Processes 23(3): 211-236.

5. Ferrari B, Moreno R (2010) A review: EPD kinetics. J Eur Ceram Soc 30, 1069-1078.

6. Sarkar P, Haung X, Nicholson PS (1993) Ceramic Engineering and Science Proceedings. The American Ceramic Society, USA, pp. 707716.

7. Van der Biest OO, Vandeperre Luc J (1999) Electrophoretic deposition of materials. Annu Rev Mater Sci 29: 327-352.

8. Bermejo R, Pascual J, Lube T, Danzer R (2008) Optimal strength and toughness of $\mathrm{Al}_{2} \mathrm{O}_{3}-\mathrm{ZrO}_{2}$ laminates designed with external or internal compressive layers. Journal of the European Ceramic Society 28(8): $1575-1583$.

9. Ferrari B, González S, Moreno R, Baudín C (2006) Multilayer coatings with improved reliability produced by aqueous electrophoretic deposition. Journal of the European Ceramic Society 26(1-2): 27-36.

10. Corni I, Ryan MP, Boccaccini AR (2008) Electrophoretic deposition: From traditional ceramics to nanotechnology. J Eur Ceram Soc 28(7): 1353-1367. 
11. Boccaccini AR, Zhitomirsky I (2002) Application of electrophoretic and electrolytic deposition techniques in ceramics processing. Curr Opin Solid Sate Mater Sci 6(3): 251-260.

12. Besra L, Liu M (2007) A review on fundamentals and applications of electrophoretic deposition (EPD). Progress in Materials Science 52(1): 1-61.

13. Boccaccini AR, Keim S, Ma R, Li Y, Zhitomirsky I (2010) Electrophoretic deposition of biomaterials. J R Soc Interface 7: 581-613.

14. Nold A, Zeiner J, Assion T, Clasen R (2010) Electrophoretic deposition as rapid prototyping method. Journal of the European Ceramic Society 30(5): 1163-1170.

15. Mochales C, Frank S, Zehbe R, Traykova T, Fleckenstein C, et al. (2013)
Tetragonal and cubic zirconia multilayered ceramic constructs created by EPD. The Journal of Physical Chemistry B 117(6): 1694-1701.

16. Fleckenstein C, Mochales C, Frank S, Kochbeck F, Zehbe R, et al. (2014) Tetragonal and cubic zirconia multilayered ceramics: investigation of electrical parameters during automated EPD processing. Advances in Applied Ceramics 113(1): 35-41.

17. Moritz T, Eiselt W, Moritz K (2006) Electrophoretic deposition applied to ceramic dental crowns and bridges. J Mater Sci 41: 8123-8129.

18. Oetzel C,Clasen R (2006) Preparation of zirconia dental crowns via electrophoretic deposition. J Mater Sci 41: 8130-8137.

19. Scott HG (1975) Phase relationships in zirconia-yttria system. J Mater Sci 10: 1527-1535. 